\title{
Vaccine-associated Paralytic Poliomyelitis Caused by Contact Infection
}

\author{
Bungo Okuda ${ }^{1}$, Eri Uetani ${ }^{2}$ and Kensho Okamoto ${ }^{1}$
}

\begin{abstract}
We encountered an adult patient with acute anterior poliomyelitis (AAP), whose monoparesis developed 28 days after his son's immunization with oral poliovirus vaccine (OPV). Neurological and electrophysiological examinations suggested that his muscular wasting of the left lower limb was due to a lower motor neuron disorder, and magnetic resonance imaging revealed the responsible lesion in the left anterior horn at the thoracolumbar junction. His stool was found to include poliovirus type 3, mainly originating from Sabin 3 by neutrization antibody and PCR-restriction fragment length polymorphism method. This indicated that the AAP resulted from contact with his son. This patient raises the question about OPV in polio-free countries.
\end{abstract}

Key words: acute anterior poliomyelitis, adult, contact infection, magnetic resonance imaging, vaccination

(DOI: 10.2169/internalmedicine.45.1622)

\section{Introduction}

Since the advent of vaccination, the incidence of acute anterior poliomyelitis (AAP) has drastically decreased in developed countries. Although oral poliovirus vaccine (OPV) as well as inactivated poliovirus vaccine (IPV) contributed to the ongoing process of global eradication of wild polioviruses, the problem of vaccine-associated paralytic poliomyelitis (VAPP) persists in OPV-using countries $(1,2)$. Illustrating this, we report on an adult patient who developed AAP following vaccination of his son with OPV. As far as we know, lesion sites of contact-induced VAPP have scarcely been revealed by magnetic resonance imaging (MRI). Some problems regarding OPV are discussed.

\section{Case Report}

A 36-year-old man presented with a 16-day history of acute monomelic paralysis. The patient had been completely healthy until November 25, 2003 (day 1), when he developed weakness of the left leg after flu-like symptoms. He subsequently had difficulty lifting the left thigh and putting on his shoes. His son at age six months had received administration of OPV 28 days prior to the onset of his pa- ralysis. Because MRI of the brain and lumbar spine in another hospital revealed no lesion responsible for the symptoms, he was referred to our department on December 11, 2003. Neurological examination on admission showed muscle weakness of the left lower limb; muscle strength of the affected limb on the Medical Research Council grade was 4/ 5 in the iliopsoas and quadriceps femoris muscles, and 3/5 in the hamstrings, tibialis anterior, and gastrocnemius muscles. There was mild muscle atrophy in the left thigh and leg with a high arch of the foot (Fig. 1). Deep tendon reflexes were generally brisk except for diminished left ankle jerk. No pathological reflex was present. Superficial and deep sensations were intact. He walked unaided with steppage gait on the left side. Lumbar puncture revealed eight mononuclear cells $/ \mathrm{mm}^{3}$, protein of $107 \mathrm{mg} / \mathrm{dl}$, and glucose of $70 \mathrm{mg} / \mathrm{dl}$. Conduction velocities of the peripheral nerves were within normal limits, but $\mathrm{F}$ wave occurrence rate of the left peroneal nerve was decreased (43\%). Needle electromyography revealed polyphasic motor unit potentials of long duration (1-2.5mV, 10-15 msec, 5-7 phases) with positive sharp waves in the left quadriceps femoris and tibialis anterior muscles. T2-weighted MRI on day 22 revealed high signal intensity in the anterior spinal cord at the level of $\mathrm{T}$ 12 and L1 (Fig. 2A), corresponding to the left anterior horn (Fig. 2B). Since the clinical features suggested AAP, we

\footnotetext{
${ }^{1}$ Department of Neurology, Ehime Prefectural Central Hospital, Matsuyama and ${ }^{2}$ Department of Geriatrics and Neurology, Ehime University Graduate School of Medicine, Toon

Received for publication October 26, 2005; Accepted for publication January 7, 2006

Correspondence to Bungo Okuda, Department of Neurology, Ehime Prefectural Central Hospital, Matsuyama 790-0024
} 


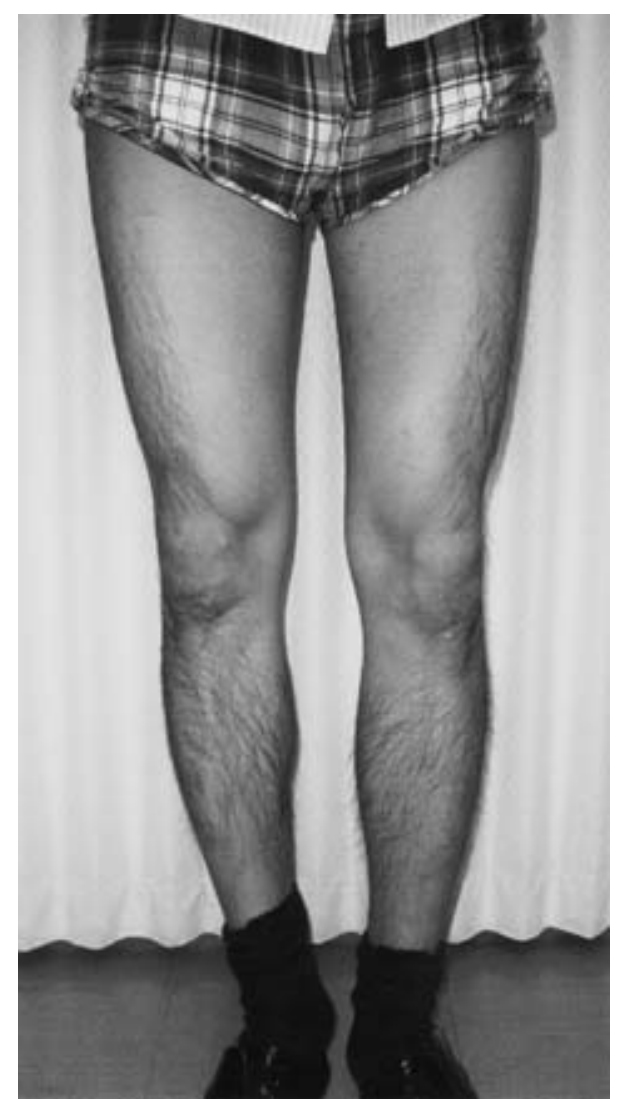

Figure 1. Photograph shows muscle atrophy in the left lower limb.

searched for evidence of infection with enteroviruses or herpesviruses by culturing cerebrospinal fluid (CSF) and by measuring antibody titers in blood and CSF cerebrospinal fluid. No evidence was detected. From his stool obtained on day 15 , however, a poliovirus was detected, which was identified as type 3 in virus neutralization test. It was found to be Sabin 3 vaccine virus, because its serotype and intratypic differentiation were similar to OPV by the PCR-restriction fragment length polymorphism method applied to the VP1 region $(3,4)$. He had been incompletely immunized in childhood with only one dose of OPV ingestion (usually three doses), which may have contributed to the occurrence of VAPP. The poliovirus came to be undetectable in his stool on day 51. During a 2-year follow-up, his monomelic weakness remained unchanged.

\section{Discussion}

This patient developed acute monomelic weakness. Neurological diseases causing acute monoparesis of the lower limb include Guillain-Barré syndrome, mononeuritis, lumbar canal stenosis,vascular lesions of the spinal cord, and myelitis of various origins. From neurological and electrophysiological examinations, his monomelic weakness was attributable mainly to a lower motor neuron disorder. Although viral myelitis involving the anterior horn was suspected, viral studies were negative, including sera and CSF culture for enteroviruses and herpesviruses. However, analysis of his stool indicated that the isolated poliovirus was vaccinederived and that his monoparesis arose from contact with his son's feces including poliovirus (4). In addition, MRI revealed clearly the responsible lesion corresponding to the anterior horn ipsilateral to the affected limb. The high intensity on MRI corresponded to the epiconus which includes motor neurons between L4 and S2. Thus, the MRI findings were compatible with the patient's muscle wasting in the lower limb. In good agreement with cinical features, the neuroimaging favored the diagnosis of VAPP.

Poliovirus is an enterovirus transmitted mainly by fecaloral contamination. After polio infection, the majority of people are asymptomatic or have mild febrile illness, whereas only one to two per cent of infected individuals develop AAP. It still remains unclear whether poliovirus involves the central nervous system by the blood stream or by way of the peripheral neural tissues (5). Although poliovirus can involve any structure of the nervous system, the selective affinity of the virus for large motor neurons exists, particularly in the anterior horn in the spinal cord and motor nuclei in the medulla. Once polio enters the central nervous system, it spreads rapidly along neural pathways, leading to flaccid paralysis, bulbar palsy, or dysautonomia. In addition to acute inflammatory tissue damage, late progression of muscle wasting or aching can occur manifesting as postpolio syndrome or motor neuron disease $(6,7)$.

Following the induction of immunization by OPV, AAP has effectively disappeared in countries with a high OPV coverage rate. On the other hand, outbreaks of AAP have been reported in this century to be associated with circulating vaccine-derived poliovirus or its recombinant strains in tropical developing countries $(8,9)$. Between 1990 and 2001, there were 11 cases of AAP in Japan. All were vaccine-associated, and did not result from wild poliovirus. In eight children, muscle wasting followed ingestion of OPV. Three adult patients developed paralytic poliomyelitis by contact infection from asymptomatic vaccine recipients in the patient's family (10). Although it remains uncertain how poliovirus reaches the nervous system, the inconsistent regional occurrence of VAPP indicates that individual variations in vulnerability to poliovirus exist in the human nervous system. This was a new patient with VAPP by contact infection who uniquely demonstrated the responsible lesion on MRI. As seen in our patient, individuals with unknown or incomplete vaccination history may be prone to develop contact paralysis (11). This case serves as a warning to clinicians to beware of this condition. Caregivers can develop VAPP by contact with recipients of OPV within 60 days after vaccination (11). The alternate choice is to use IPV, which is safe but more costly. Countries that have eliminated wild polioviruses should consider switching to IPV despite its high cost, since VAPP can be avoided. Many countries such as the USA, Canada, most of Europe, and New Zealand have adopted this route (2). 

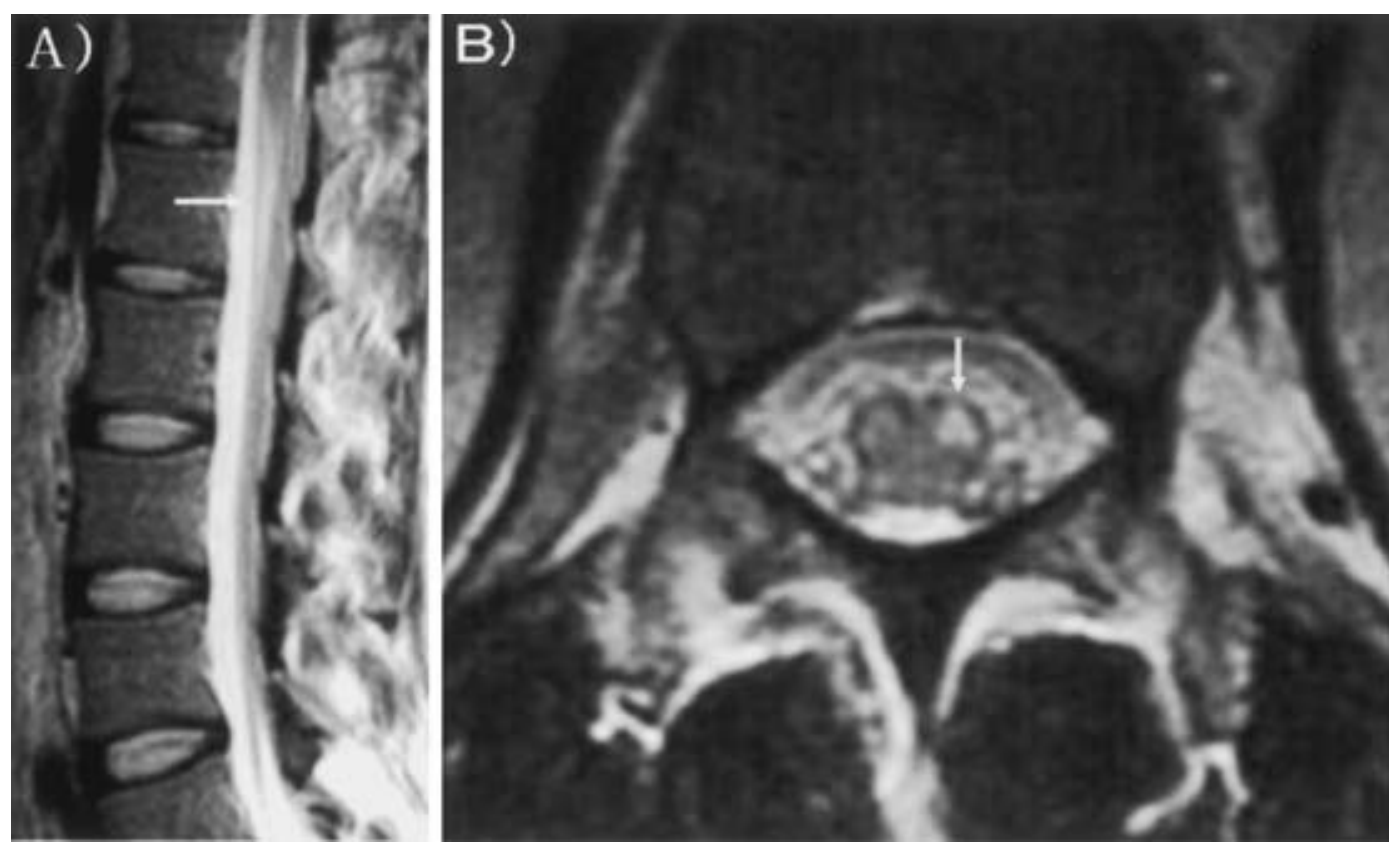

Figure 2. T2-weighted MRI reveals high intensity signal in the anterior spinal cord at the level of T12 and L1 (A, sagittal image), and in the left anterior horn at the level of T12 (B, right/left reversed axial image).

\section{References}

1. Katz SL. From culture to vaccine-Salk and Sabin. N Engl J Med 351: 1485-1487, 2004.

2. Alexander LN, Seward JF, Santibanez TA, et al. Vaccine policy changes and epidemiology of poliomyelitis in the United States. JAMA 292: 1696-1701, 2004.

3. Balanant J, Guillot S, Candrea A, Delpeyroux F, Crainic R. The natural genomic variability of poliovirus analyzed by a restriction fragment length polymorphism assay. Virology 184: 645-654, 1991.

4. Yoneyama T, Sakae K, Baba J, et al. Surveillance of poliovirusisolates in Japan, 2000. Jpn J Infect Dis 54: 80-82, 2001.

5. Donaghy M. The motor neuron diseases. In: Brain's Diseases of the Nervous System. 11th ed. Donaghy M, Ed, Oxford, New York, 2001: 443-460.

6. Jubelt B, Lipton HL. ALS: persistent scientists do not find persisting enteroviruses. Neurology 62: 1250-1251, 2004.
7. Sorenson EJ, Windebank AJ. Incidence of adaptive equipment use in subjects with a remote history of paralytic poliomyelitis. Neurology 65: 963, 2005.

8. Kew O, Morris-Glasgow V, Landaverde M, et al. Outbreak of poliomyelitis in Hispaniola associated with circulating type 1 vaccine-derived poliovirus. Science 296: 356-359, 2002.

9. Rousset D, Rakoto-Andrianarivelo M, Razafindratsimandresy R, et al. Recombinant vaccine-derived poliovirus in Madagascar. Emerg Infect Dis 9: 885-887, 2003.

10. Yoneyama T, Sasagawa A, Kikuchi M, et al. Surveillance of poliovirus-isolates in Japan, 2001. Jpn J Infect Dis 55: 57-58, 2002.

11. WHO Consultative Group. The relation between acute persisting spinal paralysis and poliomyelitis vaccine-results of a ten-year enquiry. Bull World Health Organ 60: 231-242, 1982.

(C) 2006 The Japanese Society of Internal Medicine http://www.naika.or.jp/imindex.html 